\title{
LOW-DOSE ELECTRON DIFFRACTION OF CATALASE CRYSTALS DRIED WITHIN A MATRIX OF THE DISACCHARIDE, TREHALOSE: IS A "DRY PROTEIN CRYSTALLOGRAPHY" FEASIBLE?
}

\author{
William H. Massover
}

Department of Cell Biology \& Molecular Medicine, UMDNJ - New Jersey Medical School, Newark, N.J. 07103, USA

Certain sugars can preserve native protein structure despite drying, freezing, or heating [1]. For transmission electron microscopy (TEM) and diffraction studies of protein specimens, the use of these sugar sustains as a protective matrix has been very valuable in electron crystallography of 2-D monolayer crystals with cryo-TEM [2]. The disaccharide, trehalose, has provided several protein structures at very high resolution, and its benefits for TEM use are being examined [e.g., 3-5]. It is not known whether this structure-preserving sugar can yield high resolution data for thin 3-D protein crystals. This report gives low-dose electron diffraction results with single-crystals of the enzyme, catalase, prepared within a matrix of trehalose.

Test specimens of thin platelets of bovine liver catalase were dried within an aqueous solution of trehalose (Sigma), using a standardized on-grid protocol as described [6]. Low-dose selected-area electron diffraction patterns from single-crystals at room temperature $(100 \mathrm{kV}$; JEOL $100 \mathrm{CX}$ ) [7] were recorded onto SO-163 film (Kodak) using a routine exposure time of 2 minutes, followed by development in undiluted D-19 (Kodak) for 12 minutes.

Thin catalase crystals dried within $100 \mathrm{mM}$ trehalose do not show any periodic structure by direct imaging, even using large amounts of defocus. With low-dose electron diffraction, trehalose treatment readily permits recording of single-crystal spot patterns extending into the high-angle range (Fig. 1). Use of water in place of this sugar does not yield any Bragg diffraction spots. The highest angle spots thus far recorded with trehalose are on the b-axis at the 43rd order, corresponding to periodic information for $4.1 \AA$ (Fig. 1).

These diffraction results establish that an amorphous trehalose matrix preserves the protein structure of dried thin 3-D catalase crystals into the high resolution range. This capability notably does not depend upon cooling the dried specimens to a cryogenic temperature; the structural preservation observed thus must be due in part to resistance by this sugar against the denaturing effects of drying.

It should be recognized that these specimens of catalase crystals are embedded in a light-atom matrix and therefore must be considered to be "unstained". This is verified by the inability to obtain any images showing lattice periodicities. Thus, the Bragg spot intensities are nearly entirely due to constituents of the polypeptides within the ordered unit cells. Accordingly, it now becomes reasonable to ponder the hitherto unthinkable question, is a "dry protein crystallography" feasible? By "dry", it is meant that the crystals are contained neither in mother liquor, as with x-ray diffraction, nor in a frozen-hydrated state, as with cryo-TEM; moreover, the specimens necessarily 
are exposed to the high vacuum inside the microscope column before diffraction is conducted. It certainly remains possible that these protein crystals still are not completely dry. Further research is needed to determine whether the high-angle electron diffraction data from dried catalase crystals can be processed to yield a valid reconstruction of protein structure [8].

\section{References --}

[1] J.H. Crowe, et al., Ann. Rev. Physiol. 69 (1998) 73.

[2] R.M. Glaeser, J.Struct.Biol. 128 (1999) 3.

[3] J.R. Harris, et al., J. Microsc. Soc. Am. 2 (1996) 43.

[4] S. DeCarlo, et al., J. Microsc. (Oxf.) 196 (1999) 40.

[5] T. Hirai, et al., J. Electron Microsc. 48 (1999) 653.

[6] W.H. Massover and P. Marsh, Ultramicrosc. 85 (2000) 107.

[7] W.H. Massover, et al., Ultramicrosc. 90 (2001) 7.

[8] This research was supported in part by NIH research grant GM57948 (USPHS, DHHS).

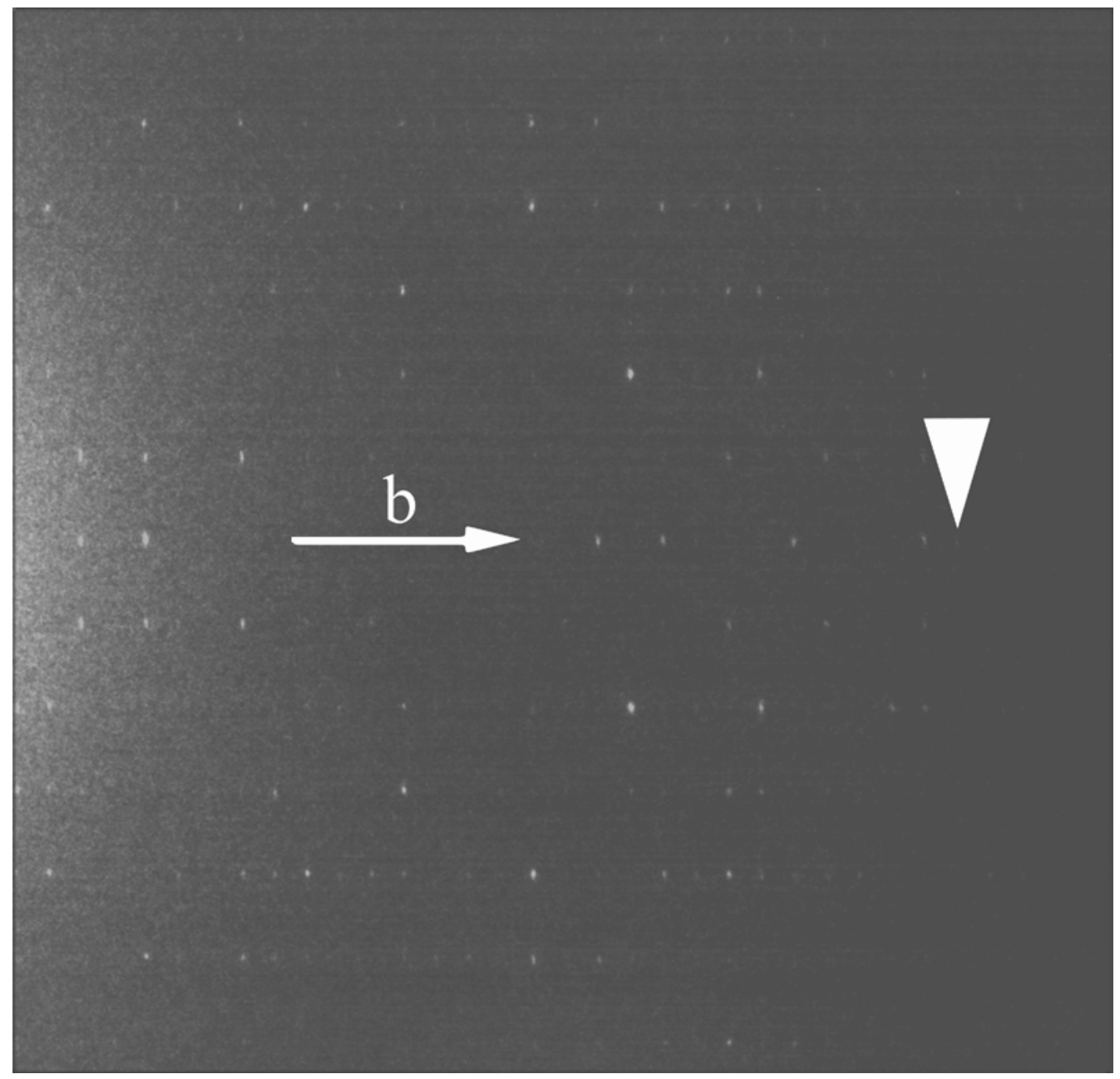

FIGURE 1. Portion of low-dose selected area diffraction pattern from a single-crystal of catalase dried within a matrix of $100 \mathrm{mM}$ trehalose. Bragg spots extend out to the $43^{\text {rd }}$ order (arrowhead) on the b-axis of the orthorhombic lattice, corresponding to a spacing of $4.1 \AA$. 